\title{
Efectos de la terapia cognitivo-conductual en factores asociados a la obesidad: un estudio de caso
}

\author{
Effects of cognitive-behavioral therapy \\ on factors associated to obesity: A case study
}

\author{
Rocío Ivonne De la Vega Morales y Liliana Cruz Hernández ${ }^{1}$
}

\begin{abstract}
RESUMEN
La obesidad es un problema mundial de salud, multifactorial, en el que intervienen factores psicológicos, conductuales y emocionales. La intervención con la terapia cognitivo-conductual parece ser una herramienta confiable para su tratamiento, por lo que bajo este enfoque se trabajó con una mujer de 62 años, con obesidad tipo I, durante 28 sesiones de forma semanal en un periodo de nueve meses. Se describen los factores involucrados en el problema y el proceso terapéutico, consistente en psicoeducación, modificación conductual, reestructuración cognitiva, emociones, habilidades sociales y resolución de problemas Los resultados mostraron un establecimiento y mantenimiento del plan alimenticio, horarios adecuados en el ciclo sueño-vigilia, manejo apropiado de las emociones relacionadas con la alimentación, una disminución de la deseabilidad social, mejoría en la percepción de la imagen corporal y aumento de habilidades en la solución de problemas, reflejada en la comunicación familiar y con el grupo social. Por lo anterior, se concluye que la terapia cognitivo-conductual es un enfoque que permite abordar de forma integral los factores asociados a la obesidad.
\end{abstract}

Palabras clave: Terapia cognitivo-conductual; Factores asociados a la obesidad; Manejo de la dieta; Imagen corporal.

\begin{abstract}
Obesity is a multifactorial worldwide health problem that involves genetic, psychological, behavioral, and emotional factors. Therapeutic interventions such as cognitive-behavioral therapy seem to be a reliable tool for its treatment. This intervention was applied to a 62-year-old woman with level I obesity, through 28 weekly sessions during a 9-month period. The therapeutic process consisted of psychoeducation, behavioral modification, cognitive restructuring, emotion regulation skills training, social skills, and problem solving. Results indicated improvement in setting up and maintaining a dietary plan, appropriate waking-sleep schedules, management of emotions related to diet, decreased undue social desirability, improved self body image perception, and problem solving skills. These changes also reflected better communication with family and friends. In conclusion, in this case, cognitive-behavioral therapy proved a psychological intervention allowing for a comprehensive approach to deal successfully with the multiple factors associated to obesity.
\end{abstract}

Key words: Cognitive-behavioral therapy; Factors associated to obesity; Intake management; Body image.

\footnotetext{
${ }^{1}$ Universidad Nacional Autónoma de México, Facultad de Psicología. Av. Universidad, 3004, Col. Copilco Universidad, Del. Coyoacán, 04510 Ciudad de México, México, tels. cels: (55)17-05-87-06 y (55)45-18-53-57, correos electrónicos: drarocioivonnedelavega@gmail.com y chl_tlsp@hotmail.com. Artículo recibido el 9 de mayo y aceptado el 1 de agosto de 2017.
} 


\section{INTRODUCCIÓN}

$\mathrm{L}$ a obesidad es una enfermedad de etiología multifactorial de curso crónico que involucra aspectos genéticos y ambientales y el estilo de vida (Cervera, Campos, Rojas y Rivera, 2010). De acuerdo con la World Health Organization (2016), se define como la acumulación anormal o excesiva de grasa que es dañina para la salud. Uno de los parámetros que hay para evaluar si una persona tiene obesidad es el índice de masa corporal (IMC), el cual se obtiene mediante la fórmula $\mathrm{kg} / \mathrm{m}^{2}$; esto es, el peso sobre la altura al cuadrado; así, a una persona con un IMC igual o superior a 30 se le considera obesa.

En los últimos años se ha visto la obesidad como un problema mundial de salud pública y se estima que 1,900 millones de adultos muestran sobrepeso y 600 millones obesidad (OMS, 2017). En cuanto a México, la Encuesta Nacional de Salud y Nutrición (ENSANUT) (Instituto Nacional de Salud Pública y Secretaría de Salud, 2012) estimó que en el año 2012 la prevalencia combinada de sobrepeso y obesidad era de $71.2 \%$, mientras que en 2016 ascendió a 72.5\%, esto es, siete de cada diez adultos padecen una de tales condiciones (cf. Observatorio Nacional de Enfermedades no Transmisibles [OMENT], 2016).

Por lo anterior, se considera alarmante el aumento de esta enfermedad en virtud de que contribuye como factor de riesgo de otras enfermedades, como las cerebrovasculares y coronarias, al igual que diabetes y cáncer de mama, esófago, colon, endometrio y riñón, entre otros (Barrera, Rodríguez y Molina, 2013). Ante ello, se ha intentado poner en práctica ciertas medidas al suponer que las causas principales de la obesidad es el desequilibrio entre la energía y las calorías consumidas gastadas, la mayor ingesta de comidas con alto nivel de grasas y el sedentarismo; sin embargo, estos esfuerzos no han sido suficientes debido a la etiología multifactorial de la obesidad, en la que intervienen factores económicos, culturales, sociales y psicológicos, los cuales se describen a continuación.

\section{Urbanización, cultura y sociedad}

Existe hoy día un aumento en la disponibilidad de alimentos procesados (adicionados con grasas, azúcar y sal), alta disponibilidad de comida rápida y exposición de la misma a la publicidad, así como escasa disponibilidad para la preparación de alimentos saludables. Por otro lado, la venta de productos que prometen reducir el peso y la publicidad acerca de la figura corporal ideal crean insatisfacción y estrategias orientadas a metas irreales (Barrera et al., 2013).

\section{Psicológicos (historia de vida, conducta $y$ emociones)}

En este factor se reconoce la historia de vida, en la cual la persona con obesidad tiene un bajo control sobre su ingesta debido a la asociación establecida con los cuidadores primarios y el alimento; en ocasiones aparece un proceso paradójico, el cual, cuando hay una restricción de alimento, hay un pobre control sobre la ingesta, de modo que, al fallar, la persona obesa sufre estrés, ansiedad y depresión. Respecto a los factores emocionales, la comida se emplea para atenuar el malestar y constituye un vehículo para manejar esas emociones, que normalmente la persona tiene dificultad para identificar (Bersh, 2006).

Debido a los diferentes factores que inciden en el padecimiento de la obesidad, el tratamiento va más allá del control de la ingesta y un aumento aparejado de la actividad física, lo que implica cambios permanentes en el estilo de vida de la persona, de manera que un manejo integral del problema requiere abordar aspectos educativos, médicos, nutricionales y psicológicos (Bersh, 2006).

En el campo de la psicología, la terapia cognitivo-conductual (TCC en lo sucesivo) es un tratamiento que se enfoca en establecer y mantener una conducta alimentaria adecuada, además de modificar los factores de vida del paciente que repercuten en la obesidad, en el que los elementos terapéuticos clave son, a saber: $a$ ) la motivación y la psicoeducación; $b$ ) las técnicas conductuales; c) la reestructuración cognitiva; $d$ ) la imagen corporal, la autoestima y la impulsividad, e) las demandas afectivas, la resolución de problemas o la 
sintomatología depresiva, y f) el entrenamiento y fortalecimiento de las técnicas de autocontrol y de las habilidades sociales. Lo anterior permite que el paciente descubra nuevas conductas para que, incorporándolas a su vida diaria, tenga como resultado un mayor bienestar y eficacia (Lafuente, 2011).

En diversas investigaciones se ha aplicado la TCC a pacientes obesos. Gade, Rosenvinge, Hjelmesæth y Friborg (2014), por ejemplo, empleando un diseño de experimento clínico en pacientes con obesidad mórbida que fueron sometidos a cirugía bariátrica, a un grupo se le sometió a un tratamiento habitual (apoyo nutricional y recomendaciones de actividad física) y a otro a TCC (psicoeducación enfocada en patrones conductuales de ingesta, técnicas de regulación afectiva, trabajo en casa, diario de alimentación, cogniciones y emociones que desencadenan el comer, reestructuración cognitiva, reforzamiento de conductas positivas y prevención de recaídas). Los resultados mostraron que, en comparación con los controles, los pacientes con TCC disminuyeron significativamente sus síntomas de ansiedad y depresión, además de que tuvieron una mayor pérdida de peso.

Asimismo, la efectividad de la TCC se ha demostrado a lo largo del tiempo. En un estudio de Calugi et al. (2016), pacientes con obesidad severa con y sin trastorno de atracón fueron intervenidos con este tipo de terapia (psicoeducación, etiqueta de alimentos, autocontrol, estrategias de control de estímulos, reestructuración cognitiva, resolución de problemas y prevención de recaídas). Los resultados mostraron que hubo una mejoría significativa al finalizar el tratamiento, y en un seguimiento a cinco años ambos grupos mantuvieron una pérdida de peso similar, lo que demostró que la TCC puede ser una estrategia prometedora a corto y a largo plazo.

Por lo tanto, el abordaje de la TCC ha considerado un trabajo dinámico y directivo con las variables que pueden interferir en la obesidad y ha mostrado evidencia empírica en investigaciones con grupos controlados (Larrañaga y García, 2007); sin embargo, la presentación de las variables psicológicas y conductuales no se identifican o se abordan de forma específica debido a que los estudios se han realizado empleando muestras. Por ello, el objetivo del siguiente reporte es presentar un estudio de caso en un paciente con obesidad, donde se abordaron la identificación y modificación de los factores conductuales y psicológicos mediante la estrategia terapéutica de TCC.

\section{ESTUDIO DE CASO}

La paciente AP es una mujer de 62 años que acudió a consulta de primera vez a un centro de atención psicológica perteneciente al sector público. El motivo que refirió la paciente fue el siguiente: "Tengo un sobrepeso de 25 kilos, colesterol y triglicéridos altos; peso 83 kilos y mido $1.58 \mathrm{~cm}$; generalmente como de más cuando estoy preocupada o estresada, y me gusta mucho el pan y los postres, además de la fruta". Por lo anterior, se le canalizó al programa "Terapia cognitivo-conductual en trastornos de la conducta alimentaria" de la propia institución.

Mediante una serie de entrevistas, se obtuvo la siguiente información: AP era jubilada de un empleo en recursos humanos, con nivel de maestría, que vivía con dos hijos adoptados de 25 y 19 años y un hermano de 47 años. Su hija mayor estaba casada y tenía un bebé de ocho meses, quienes también residían en el hogar de la paciente; no tenía empleo en ese momento y no participaba en los quehaceres del hogar. En cuanto a su hijo menor, este comenzó a trabajar porque no ingresó a la universidad, sin embargo, era una figura de apoyo de AP; respecto al hermano de la paciente, él tampoco laboraba y participaba míninamente en las actividades del hogar. La comunicación era escasa entre los miembros de la familia, de la cual la paciente era el sostén económico, para lo cual utilizaba su pensión de jubilación.

Los antecedentes identificados fueron un empleo de oficina a lo largo de quince años, donde la alimentación consistía en carbohidratos y grasas, sin consumo de agua ni actividad física. AP se divorció poco después, y a partir de entonces comenzó su problema de obesidad; expresó: "Me divorcié, bajé la cortina y me eché a engordar". Tal información se interpretó posteriormente en el proceso terapéutico debido a que la ingesta calórica y la falta de autocuidado hacia su imagen fue un mecanismo de afrontamiento ante el divorcio. 
Los hábitos de ingesta de la paciente se identificaron a través de sus diarios de alimentación, donde se encontró un alto consumo de carbohidratos simples (galletas y pastelillos) que acompañaba con café, nulo consumo de verduras, grasas buenas y agua; asimismo, a través de los citados diarios se identificaron estados emocionales tales como enojo, que no expresaba en virtud de que ella suponía que "siempre debía estar bien para los demás y con una sonrisa". Cada vez que se sentía enojada comía el tipo de alimentos mencionados; de igual forma, se identificaron situaciones que aumentaban su ansiedad, por ejemplo una pobre comunicación entre los miembros de la familia, falta de límites, responsabilidades distribuidas de manera inequitativa e inconformidad ante diferentes situaciones, la que no comunicaba a los demás, así como problemas económicos ya que AP jugaba el rol de líder permisivo, por lo que tenía muchas responsabilidades en el hogar que no eran valoradas positivamente por la familia, todo lo cual afrontaba mediante la ingesta de alimentos.

Los pensamientos de la paciente asociados a su peso, autoimagen, autoestima y autoconcepto se ejemplifican con afirmaciones como las siguientes: "Todos los profesionales deben verse bien y tener buena figura", "Hay alimentos que cuando están presentes se deben aprovechar (pastel)", "No puedo contradecir a los demás porque todo lo reflejo en mi peso", "Me gustaría ser una sirena"; tales afirmaciones devaluaban sus logros y a la vez le imponían altas exigencias.
Asimismo, la paciente reportó sufrir estreñimiento y problemas para conciliar el sueño. Los factores de riesgo que se identificaron fueron la socialización mediante la comida y su condición médica de hipotiroidismo.

AP había intentado aminorar su obesidad mediante diferentes dietas que encontraba en Internet; sin embargo, se sometió a un tratamiento médico a lo largo de seis meses consistente en una dieta cetogénica ${ }^{2}$ con la que bajó de peso; sin embargo, abandonó dicho tratamiento porque no quería consumir los medicamentos prescritos para adelgazar, además de que no se sentía satisfecha con su imagen corporal ya que percibía flacidez en su piel, sobre todo en el área del cuello.

Por último, la paciente comentó que el factor que la motivó para asistir a la intervención terapéutica fue la preocupación que tenía por sus hábitos alimentarios, pues no quería volver a incrementar su peso, sobre todo porque había empezado una relación de pareja y deseaba verse atractiva.

\section{MÉTODO}

Con la información recabada en las entrevistas ya citadas, se aplicó una batería de pruebas para complementar la valoración psicológica, mismas que se muestran en la Tabla 1.

Para realizar la integración de las pruebas psicológicas al diagnóstico final, se tomaron en cuenta los puntajes obtenidos que se muestran en la Tabla 2.

Tabla 1. Pruebas utilizadas para la valoración psicológica.

\section{Prueba de Actitudes hacia la Alimentación (EAT-40)}

(Garner y Garfinkel, 1979)

Consta de 40 reactivos autoaplicables que evalúan características psicológicas o conductuales compatibles con trastornos de la conducta alimentaria, los que se distribuyen en tres factores: dieta, control oral, y bulimia y preocupación por el alimento. Cada respuesta tiene valores entre 0 a 3 puntos. Fue adaptada para población mexicana por Álvarez, et al. (2004). Para medir la consistencia interna de la prueba se utilizó el índice de confiabilidad de Cronbach, obteniéndose un coeficiente alfa $(\alpha)$ de .90 en la muestra con TCA y de .93 en la muestra total. El puntaje máximo es 120 y su punto de corte es 28.

\section{Inventario de Desórdenes de la Alimentación (EDI II)}

(Garner y Olmstead, 1983)

Este inventario contiene 91 preguntas autoaplicables que evalúan características nucleares psicológicas y conductuales de los trastornos de la conducta alimentaria: 1) impulso por la delgadez, 2) consciencia interoceptiva, 3) bulimia, 4) insatisfacción corporal, 5) sensación de inutilidad, 6) temor a la madurez, 7) perfeccionismo, 8) desconfianza interpersonal, 9) ascetismo, 10) regulación de impulsos y 11) inseguridad social. En una escala tipo Likert, las opciones de respuesta oscilan de "nunca" a "siempre". La prueba fue adaptada y estandarizada para población mexicana por García, Vázquez, López y Arcila (2003) y cuenta con un $\alpha$ de .94 para mujeres con TCA. Para la interpretación de los resultados, se deben comparar los puntajes obtenidos con las medias y desviaciones estándar de los pacientes con TCA.

${ }^{2}$ Dietas bajas en hidratos de carbono, cuyo contenido suele ser inferior a 50-60 g al día (Covarrubias, Aburto y Sámano, 2013). 


\section{Cuestionario sobre Forma Corporal (BQS) \\ (Cooper, Taylor, Cooper y Fairmont, 1987)}

Contiene 34 reactivos que evalúan alteraciones actitudinales respecto a la imagen corporal durante el último mes por medio de cuatro subescalas: 1) insatisfacción corporal, 2) miedo a engordar, 3) baja estima por la apariencia y 4) deseo de perder peso. Fue adaptada para población mexicana por Vázquez et al. (2011), tiene una consistencia interna de .98 y consta de dos factores: malestar corporal normativo $(\alpha=.95)$ y malestar corporal patológico $(\alpha=.94)$. Cada pregunta tiene valores que oscilan entre 1 y 6. Su punto de corte es 105, a partir del cual se considera un problema en cuanto a la imagen corporal.

\section{Cuestionario de Actitudes hacia el Cambio en Trastornos de la Conducta Alimentaria (ACTA) \\ (Beato y Rodríguez, 2003)}

Es un cuestionario de 59 reactivos distribuidos en seis subescalas: precontemplación, contemplación, decisión, acción, mantenimiento y recaída. Refleja la fase en la que el sujeto está percibiendo su problema. El instrumento en español (Beato y Rodríguez, 2003) tiene una consistencia interna mayor de .70 y se considera el estado motivacional de la fase de cambio al dominio que cuente con mayor puntuación.

\section{Escala Visual de Figuras (EVF) \\ (García y Gómez-Peresmitré, 2003)}

Consta de nueve figuras y mide satisfacción o insatisfacción con la imagen corporal. De acuerdo con la diferencia de la identificación y el ideal visual, se interpreta el grado de satisfacción (figura actual, figura ideal), donde 0 significa que no hay insatisfacción; por el contrario, a mayor diferencia, mayor insatisfacción corporal.

Inventario de Ansiedad (BAI)

(Beck, Brown, G., Epstein, N. y Steer, 1988)

Este inventario consta de 21 reactivos de síntomas de ansiedad en una escala de 0 a 3 . Fue adaptada para población mexicana por Robles, Varela, Jurado y Páez (2001). El análisis factorial de componentes principales con rotación varimax arrojó cuatro factores principales. Su índice de confiabilidad es de .83. La norma de calificación para población mexicana es la siguiente: Mínima: 0-5, Leve: 6-15, Moderada: 16-30, Severa: 31-63.

\section{Inventario de Depresión de Beck (BDI) \\ (Beck y Steer, 1996)}

Cuenta con 21 reactivos autoaplicables y mide el factor cognitivo-conductual y el factor somático en una escala de cuatro puntos, cuya suma total fluctúa entre 0 y 63 puntos. Fue adaptada por Jurado et al. (1998) para población mexicana, obteniéndose un $\alpha$ de 0.87. Las normas para la población general son, a saber: 0-9, mínima; 10-16, leve, 17-29, moderada y 30-63, severa.

Tabla 2. Resultados cuantitativos obtenidos en las pruebas.

\begin{tabular}{|l|l|c|}
\hline Prueba & \multicolumn{1}{|c|}{ Subescalas } & Puntajes obtenidos \\
\hline EAT-40 & \multicolumn{1}{|c|}{ 1) Impulso por la delgadez } & $33^{*}$ \\
\hline \multicolumn{5}{|l}{ EDI II } & 2) Consciencia interoceptiva & $16^{*}$ \\
& 3) Bulimia & $8^{*}$ \\
& 4) Insatisfacción corporal & $1 *$ \\
& 5) Sensación de inutilidad & $17^{*}$ \\
& 6) Temor a la madurez & $0^{*}$ \\
& 7) Perfeccionismo & $6^{*}$ \\
& 8) Desconfianza interpersonal & $13^{*}$ \\
& 9) Ascetismo & $0^{*}$ \\
\hline BSQ & 10) Regulación de impulsos & $5^{*}$ \\
\hline EVF & 11) Inseguridad social & 0 \\
\hline \multirow{5}{*}{ ACTA } & 1) Precontemplación & 3 \\
& 2) Contemplación & $130^{*}$ \\
& 3) Decisión & 3 \\
\hline BAI & 4) Acción & $29 *$ \\
\hline BDI & 5) Mantenimiento & $27^{*}$ \\
\hline
\end{tabular}

*Pruebas con puntaje significativo. 


\section{Interpretación de los resultados de las pruebas psicológicas}

Mediante el análisis de los resultados, se observó que en la prueba EAT-40 hubo actitudes y pensamientos negativos relacionados con la conducta alimentaria; el análisis cualitativo mostró una dieta poco variada, lo cual se corroboró posteriormente en el autorregistro, que es un formato en el cual el paciente describe qué come, en dónde, con quién y qué pensamientos y sentimientos tiene al momento de dicho consumo. Respecto a la prueba EDI-II, se pudo advertir tanto un impulso a la delgadez como una marcada insatisfacción corporal, así como también un alto nivel de perfeccionismo; esto último se manifestó en reactivos como: "empeñarse en no decepcionar a los otros o cumplir las exigencias" y por último se apreció un nivel alto de ascetismo, el cual se vinculó con reactivos que mostraban una búsqueda de autocontrol sobre sus sensaciones corporales.

Ahora bien, en las pruebas de imagen corporal (BQS y Escala Visual de Figuras) los resultados mostraron insatisfacción corporal; en primer lugar, en el BQS la paciente manifestó sentimientos negativos cuando se miraba a un espejo, así como una percepción distorsionada al afirmar "verse" más grande después de cada comida; asimismo, creía que su figura corporal era el resultado de su falta de autocontrol; en segundo lugar, dentro de la Escala Visual de Figuras señaló una percepción cercana a la que tenía, pese a lo cual le gustaría ubicarse en una imagen corporal dos escalas más abajo a la que correspondía (Figura 1).

Figura 1. Percepción corporal de AP: (D) indica cómo se percibía y (A) cómo quería verse.

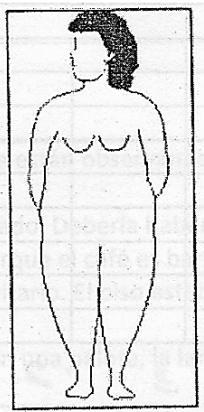

(D)

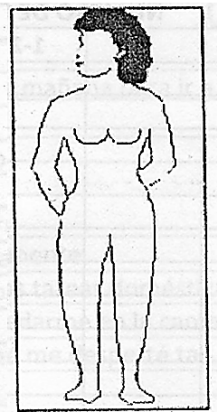

(A)
Tomado de Escala Visual de Figuras (García y Gómez-Peresmitré, 2003).
Respecto a la motivación al cambio (ACTA), la paciente mostró resultados favorables, encontrándose en el estado de decisión, lo que se relacionó con la preocupación que tenía por sus hábitos de alimentación y el modo en que esto podría afectar su salud, reconociendo que le costaba un trabajo considerable cambiar su conducta, aunque estaba dispuesta a esforzarse para lograrlo.

Los instrumentos complementarios de ansiedad y depresión no indicaron la presencia de dichos estados. Específicamente en la escala de ansiedad, se encontró una discrepancia dado que la paciente reportó que su sobreingesta estaba ligada a sentimientos de preocupación o ansiedad, por lo que se supone que los factores cognitivos y somáticos de la prueba no fueron sensibles para discriminar adecuadamente este rubro.

\section{Impresión diagnóstica}

Se estableció como diagnóstico una obesidad tipo I $(\mathrm{IMC}=33.24)$, ligada a una combinación de factores médicos, como el hipotiroidismo; el estilo de vida, la falta de educación alimentaria, la ingesta como una forma de afrontamiento a situaciones que se le dificultaba expresar -como la falta de asertividad y comunicación de su enojo-, un nivel alto de ansiedad que se disminuía al consumir alimentos dulces, lo que se consideró como una ingesta hedónica, así como también problemas de autoestima y autoconcepto, dado el rol de AP en la familia, que funcionaba como un pilar resolutivo de problemas que eran ajenos a ella, por lo que era una fuente a la que constantemente recurría su familia, a la cual la paciente apoyaba sin recibir nada a cambio. Igualmente se encontraron distorsiones cognitivas respecto a su imagen corporal, un locus de control externo y un sistema de "deber ser" alto; esto es, la opinión de los demás validaba más su estado de ánimo que su bienestar personal, por lo que las habilidades para manejar sus sentimientos y la asertividad eran poco adaptativos.

\section{Plan de tratamiento}

De acuerdo al diagnóstico emitido, se fijaron los siguientes objetivos que se trabajaron bajo el enfoque de la TCC: 1) establecimiento y mantenimiento de un plan de alimentación adecuado, indicado por 
un nutriólogo titulado y con diplomado en problemas de obesidad; 2) modificación ambiental mediante diferentes estrategias conductuales; 3) reestructuración de las distorsiones cognitivas y de los conflictos de pensamiento relacionados con su imagen corporal y baja autoestima; 4) manejo de las emociones que interferían con su ingesta alimentaria, y 5) habilidades sociales, comunicación efectiva, asertividad y solución de problemas, mismos que se fueron modificando y adaptando conforme se avanzó en el proceso (Tabla 3).

Tabla 3. Proceso detallado de la intervención terapéutica.

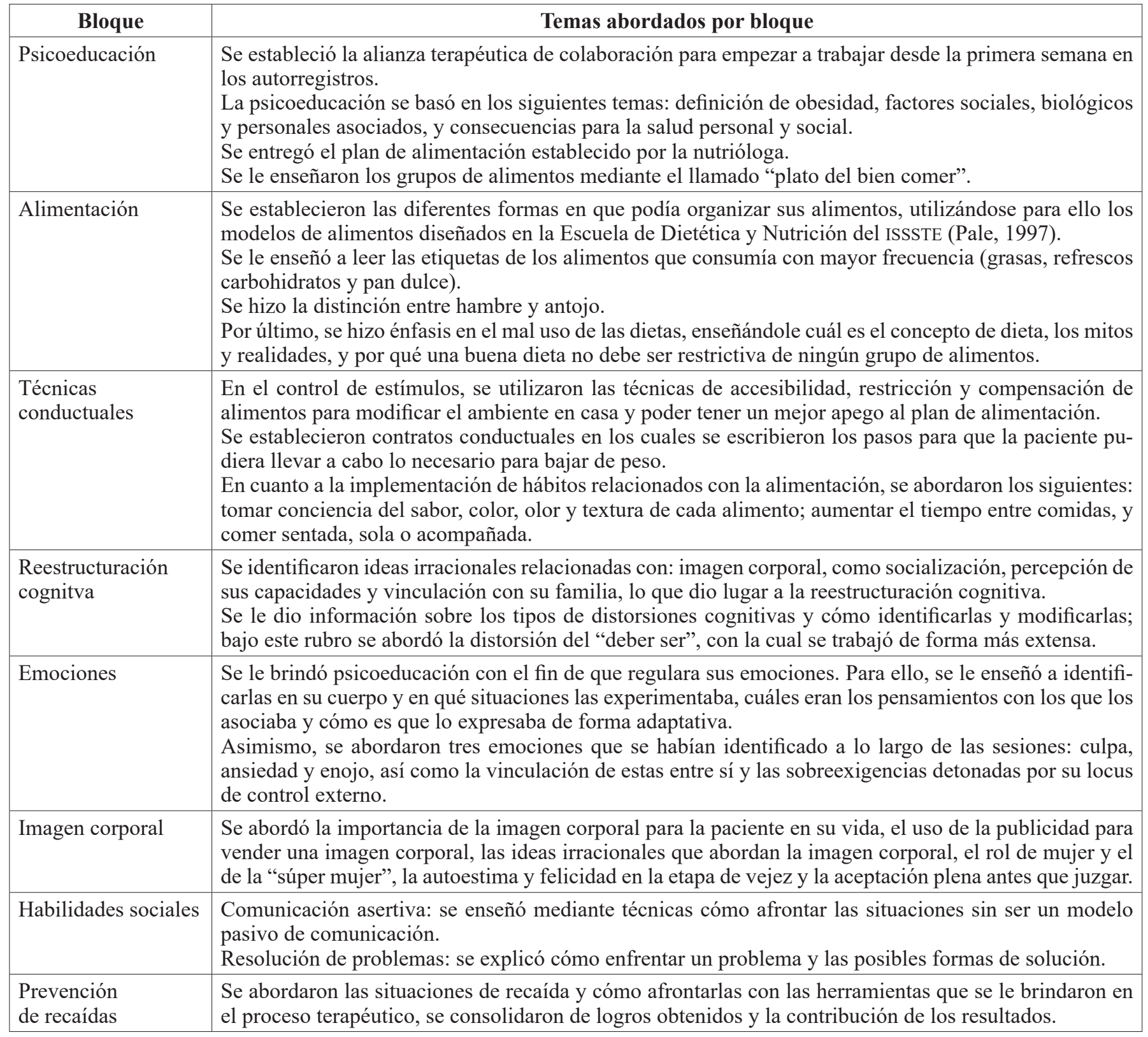

\section{RESULTADOS}

De acuerdo a los objetivos establecidos, AP tuvo una consulta psicológica semanal con duración de 60 minutos. En total fueron 28 sesiones terapéuticas. A partir de la novena sesión, la paciente se apegó a su plan de alimentación en $80 \%$, y a partir de la vigésima logró hacerlo a 100\%. En cuanto al peso, al comienzo del tratamiento la paciente pesaba 83 kilos y al final 85 (IMC $=34.04$, Obesidad I), glucosa normal (87 mg/dL), colesterol alto (267 mg/dL) y triglicéridos altos (322 mg/dl). 
En las primeras dos fases, los resultados de la intervención que se obtuvieron fueron el cambio y mantenimiento de una dieta variada (Gráfica
1) y el establecimiento de hábitos saludables, conciencia de alimentación e identificación adecuada de alimentos.

Gráficas 1 y 2. Alimentación pre y post a la intervención TCC.
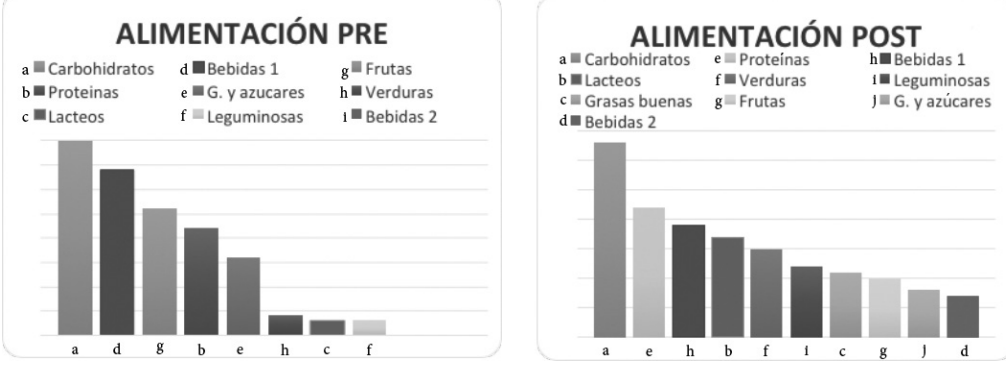

Gráfica 1: Eje horizontal: se muestran los grupos alimenticios, clasificados de acuerdo a la Escuela de Dietética y Nutrición del ISSSTE. Eje vertical: Frecuencia de consumo, considerando los primeros 15 y los últimos 15 días de tratamiento. Bebidas 1: Se refiere al consumo de líquidos agregados con azúcar: tés, refrescos, café, aguas de sabor o jugos. Bebidas 2: Líquidos libres de azúcar, agua simple. Grasas buenas: Consumo de almendras, nueces, aceite de oliva o aguacate.

Asimismo, las técnicas conductuales permitieron la adecuación ambiental y la sustitución de alimentos; además, al seguir la técnica de control de estímulos en la accesibilidad de alimentos, la paciente implementó contenedores de verdura picada con el propósito de ingerirlos a lo largo de la semana; asimismo, aumentó el tiempo de comida; por último, los contratos conductuales ayudaron a mejorar su calidad de sueño.

En cuanto a la reestructuración cognitiva, se trabajó con los pensamientos más recurrentes, como "no puedo contradecir a los demás porque todo lo reflejo en mi peso" y las distorsiones cognitivas del "deber", en las cuales reflejaba su perfeccionismo, autoexigencia y locus de control externo. Los logros identificados fueron la reducción de pensamientos negativos y el establecimiento de límites con otras personas. Respecto al manejo de emociones, se logró una identificación de las reacciones viscerales y las situaciones en que ocurrían (por ejemplo, dijo sentir corporalmente la ansiedad en el estómago, en la falta de respiración y dolor de cabeza, y en situaciones en las que no podía tener el control), al igual que su expresión adecuada de sus estados emocionales en cuanto a la culpa sin relacionarlos con su conducta alimentaria. AP pudo reducir sus exigencias hacia ella misma y a la búsqueda de aprobación. En lo tocante a la ansiedad, se promovió la cooperación de todos los miembros de la casa, y asimismo se le enseñaron técnicas de relajación y la identificación de pensamientos automáticos que la llevaban a manifestarla; en lo relativo al enojo, mediante el juego de roles logró expresarlo de forma adaptativa.

En la imagen corporal se logró que AP visualizara el cuerpo en función, antes que la estética, del autocuidado y el respeto por medio de una "carta a su cuerpo", y hubo una reconciliación con ella misma, con reconocimiento y aceptación de su persona.

Por último, los logros encontrados en cuanto a sus habilidades sociales consistieron en una comunicación eficaz con los miembros de su familia, por lo que pudo exponer lo que le incomodaba y los cambios que quería realizar en su hogar; además, enfrentó el temor que tenía de tratar algunos temas con las personas con las que trabajaba directamente en la asociación, lo que le generaba ansiedad; en cuanto a la solución de problemas, hubo una considerable respuesta ante el manejo de la técnica.

Finalmente, para prevenir recaídas la paciente retomó el deporte y buscó otras actividades para cuando dejara su cargo en la asociación en la que participaba. La comunicación con su familia mejoró de forma significativa, en especial con su hermano e hijo, lo que se tradujo en cambios en beneficios de todos. 


\section{DISCUSIÓN}

Los resultados obtenidos consistieron en el mantenimiento de la dieta, la organización de alimentos y rutinas, el entrenamiento, manejo y expresión de emociones, una comunicación familiar asertiva, la regulación de ansiedad y la resolución de problemas, por lo que se puede decir que la terapia cognitivo-conductual-además de tratar enfermedades como la obesidad- puede representar un abordaje adecuado en el que se consideran los objetivos del paciente, se evalúan los factores que intervienen en la conducta de ingesta, se trabaja la estructuración ambiental, se consideran los pensamientos y emociones y se brinda al paciente herramientas suficientes, cognitivas como emocionales, de forma personalizada y realista y con alta efectividad, para adquirir herramientas y buscar alternativas más adaptativas y viables en la vida cotidiana, debido a que la TCC enfocada en la obesidad tiene como pilares el automonitoreo, el control de estímulos, la flexibilidad en el programa de alimentación, la reestructuración cognitiva, el manejo de las relaciones interpersonales y del estrés, la motivación, los cambios de actitud al comer y la prevención de recaídas, lo contribuye, a diferencia de otros tratamientos, a evitar el abandono de la psicoterapia (Velásquez y Alvarenga, 2001).
La nula disminución de peso de la paciente y sus niveles aún altos de triglicéridos y colesterol se atribuyen a su hipotiroidismo, que fue una variable que influyó debido a que, como se sabe, en un paciente con hipotiroidismo se asocia con hipercolesterolemia, así como con una mayor viscosidad sanguínea, por lo que hay una mayor dificultad para bajar de peso y reducir los citados niveles en sangre (Cuevas y Pérez, 2006).

En conclusión, el presente trabajo mostró el análisis de un estudio de caso, en donde se expone la eficacia del TCC, originalmente enfocado a la obesidad, en otros ámbitos psicológicos y sociales, por lo que se considera que es una herramienta efectiva de tratamiento que debe reconocerse dentro del campo de la psicología y de la nutrición, en un trabajo multidisciplinario.

Como limitaciones del estudio, se reconoce la falta de un examen previo en sangre (glucosa, triglicéridos y colesterol), lo que impide comparar dichos datos clínicos antes y después del tratamiento, además de la condición médica de hipotiroidismo de la paciente, que impacta en los resultados del estudio; sin embargo, por el tipo de intervención no se podría considerar un efecto negativo; además debido a la estructura del programa, no se pudieron realizar sesiones de seguimiento para observar el mantenimiento de los resultados.

\section{REFERENCIAS}

Álvarez R., G., Mancilla D., J.M., Vázquez A., R., Unikel, C., Caballero R., A. y Mercado C., D. (2004). Validity of the Eating Attitudes Test: A study of Mexican eating disorders patients. Eating and Weight Disorders-Studies on Anorexia, Bulimia and Obesity, 9(4), 243-248.

Barrera C., A., Rodríguez G., A. y Molina A., M.A. (2013). Escenario actual de la obesidad en México. Revista de Medicina del Instituto Mexicano del Seguro Social, 51(3), 292-299.

Beato F., L. y Rodríguez C., T. (2003). El Cuestionario de Actitudes frente al Cambio en los Trastornos de la Conducta Alimentaria (ACTA). Desarrollo y propiedades psicométricas. Actas Españolas de Psiquiatría, 31(3), 111-119.

Beck, A.T., Brown, G., Epstein, N. y Steer, R.A. (1988). An inventory for measuring clinical anxiety: Psychometric properties. Journal of Consulting and Clinical Psychology, 56, 893-897.

Beck, A.T., y Steer, R.A. (1993). Beck Depression Inventory Manual. San Antonio, TX: The Psychological Corporation.

Bersh, S. (2006). La obesidad: aspectos psicológicos y conductuales. Revista Colombiana de Psiquiatría, 35(4), 537-546.

Calugi, S., Ruocco, A., El Ghoch, M., Andrea, C., Geccherle, E., Sartori, F. y Dalle Grave, R. (2016). Residential cognitive-behavioral weight-loss intervention for obesity with and without binge-eating disorder: A prospective case-control study with five-year follow-up. International Journal of Eating Disorders, 49(7), 723-730.

Cervera S., B., Campos N., I., Rojas, R. y Rivera, J. (2010). Obesidad en México: epidemiología y políticas de salud para su control y prevención. Gaceta Médica de México, 146, 397-407.

Cooper, P.J., Taylor, M.J., Cooper, Z. y Fairbum, C.G. (1987). The development and validation of the Body Shape Questionnaire. International Journal of Eating Disorders, 6(4), 485-494. 
Covarrubias G., P., Aburto G., M. y Sámano O., L.F. (2013). Dietas cetogénicas en el tratamiento del sobrepeso y la obesidad. Nutrición Clínica y Dietética Hospitalaria, 33(2), 98-111.

Cuevas R., D. y Pérez E., B. (2006). Disfunción tiroidea subclínica. Consideraciones diagnósticas y terapéuticas. Revista de Investigación Clínica, 58(6), 608-616.

Instituto Nacional de Salud Pública y Secretaría de Salud (2012). Encuesta Nacional de Salud y Nutrición 2012 (Adultos: Sobrepeso y Obesidad). (p.180). México: Autores.

Gade, H., Rosenvinge J.H., Hjelmesæth, J. y Friborg, O. (2014). Effectiveness of a cognitive behavioral therapy for dysfunctional eating among patients admitted for bariatric surgery: a randomized controlled trial. Obesity Facts, 7(2), 111-119.

García, M.A. y Gómez-Peresmitré, G. (2003). Insatisfacción corporal y seguimiento de dieta: una comparación transcultural entre adolescentes de España y México. International Journal of Clinical and Health Psychology, 3(1), 9-21.

García G., E., Vázquez V., V., López A., J.C. y Arcila M., D. (2003). Validez interna y utilidad diagnóstica del Eating Disorders Inventory en mujeres mexicanas. Salud Pública de México, 45(3), 206-210.

Garner, D.M. y Garfinkel, P.E. (1979). The Eating Attitudes Test: An index of the symptoms of anorexia nervosa. Psychological Medicine, 9(2), 273-279.

Garner, D.M., Olmstead, M.P. y Polivy, J. (1983). Development and validation of a multidimensional eating disorder inventory for anorexia nervosa and bulimia. International Journal of Eating Disorders, 2(2), 15-34.

Jurado, S., Villegas, M.E., Méndez, L., Rodríguez, F., Loperena, V. y Varela, R. (1998). La estandarización del Inventario de Depresión de Beck para los residentes de la Ciudad de México. Salud Mental, 21(3), 26-31.

Lafuente, D. (2011). Tratamiento cognitivo-conductual de la obesidad. Trastornos de la Conducta Alimentaria, 14, 1490-1504.

Larrañaga, A. y García M., R. (2007). Tratamiento psicológico de la obesidad. Medicina Clínica, 129(10), 387-391.

Observatorio Nacional de Enfermedades no Transmisibles (OMENT) (2016). Cifras de sobrepeso y obesidad en México 2016. Recuperado de: http://oment.uanl.mx/cifras-de-sobrepeso-y-obesidad-en-mexico-ensanut-mc-2016/.

Organización Mundial de la Salud (OMS). (2017). Obesidad. Recuperado de: http://www.who.int/topics/obesity/es/.

Pale, M.L. (1997). Modelos de alimentos para la orientación alimentaria. México: Secretaría de Salud.

Robles, R., Varela, R., Jurado, S. y Páez, F. (2001). Versión mexicana del Inventario de Ansiedad de Beck: propiedades psicométricas. Revista Mexicana de Psicología, 18(2), 211-218.

Vázquez A., R., Galán J., J., López A., X., Álvarez R., G.L., Mancilla D., J.M., Caballero R., A. y Unikel S., C. (2011). Validez del Body Shape Questionnaire (BSQ) en mujeres mexicanas. Revista Mexicana de Trastornos Alimentarios, 2(1), 42-52.

Velásquez V., V. y Alvarenga J., C.L. (2001). Psicología y obesidad. Revista de Endocrinología y Nutrición, 9(2), 91-96.

World Health Organization (2016). Obesity and overweight. Geneve: wHO. Recuperado de: http://www.who.int/mediacentre/ factsheets/fs311/en/. 